\title{
Utilisation de l'oxyde de chrome chez les vaches laitières pour la prévision des quantités de fèces émises. \\ 1. Etude des variations du taux de récupération et ses conséquences sur l'estimation de la digestibilité et des quantités ingérées de rations d'herbe et d'ensilage de maïs
}

Catherine MELIX, J. L. PEYRAUD, R. VÉRITÉ

Station de Recherches sur la Vache laitière, I.N.R.A. Saint-Gilles, 35590 L'Hermitage, France.

Summary. Faecal recovery of chromium oxide was examined in two experiments with lactating cows. Lower recovery with fresh grass $(0.952)$ than with corn silage $(0.996)$ seemed to be an experimental artefact. We have suggested using a faecal recovery of 1 in grazing experiments. Monitoring chromium oxide distribution lowered recovery variability and gave unbiased results.

L'estimation des quantités de fèces émises par la technique de dilution d'un marqueur indigestible suppose une récupération complète et peu variable du marqueur. Dans cette expérience, nous avons étudié les variations de la récupération de l'oxyde de chrome chez la vache laitière selon l'animal, la période et la ration considérés et leurs conséquences sur l'estimation de la digestibilité (dMO) et des quantités ingérées (Ql).

Matériel et méthodes. Dans un premier essai, le taux de récupération de l'oxyde de chrome (TR) a été mesuré au cours de 2 séries ( $A$ et $B$ ) de 4 périodes, sur 4 vaches laitières Pie-Noires en stalle à digestibilité, produisant $24 \mathrm{~kg}$ de lait à $4 \%$ et recevant une ration à base d'ensilage de maïs. Six mesures sur 32 n'ont pas été retenues par suite de problèmes liés à la collecte des fèces. Dans un second essai, la récupération a été mesurée au cours de 6 périodes (mai à septembre 1985) sur 4 vaches Pie-noires en stalle à digestibilité produisant $18 \mathrm{~kg}$ de lait à $4 \%$ et recevant de l'herbe verte à volonté (raygrass anglais ou dactyle). Pendant la troisième période, un échantillon individuel journalier de fèces a été prélevé pour l'analyse de la variabilité de l'excrétion du chrome. L'oxyde de chrome (en poudre dans l'essai 1 ; fixé sur poudre de cellulose dans l'essai 2) a été incorporé à raison de $2 \%$ dans un concentré distribué en 2 repas par jour $(8 \mathrm{~h}, 17 \mathrm{~h})$ pendant $10 \mathrm{j}(1,2 \mathrm{~kg} / \mathrm{j}$ dans l'essai $1 ; 1 \mathrm{~kg} / \mathrm{j}$ dans l'essai 2$)$. Les fèces ont été collectées pendant les 5 derniers jours. Les teneurs en chrome ont été déterminées par la méthode décrite par Poncet et Rayssiguier (1980).

Résultats et discussion. Le taux de récupératıon journalier de l'oxyde de chrome (TRj) est assez variable ( $\mathrm{CV}=7,8 \%)$, mais ne présente pas de différences systématiques entre jours. Ceci indique que dans nos conditions, la distribution préliminaire du chrome pendant 5 jours est suffisante pour obtenir un état d'équilibre. En accord avec les conclusions de Thill et al. (1978), la variabilité de la récupération proviendrait autant de celle des processus digestifs ( $\mathrm{CV}$ de l'excrétion fécale : $9,1 \%$ ) que de l'irrégularité du transit du chrome ( $C V$ de la teneur en chrome des fèces : $5,9 \%$ ).

Le taux de récupération mesuré sur des périodes de 5 jours (TRp) n'a pas été différent de 1 avec l'ensilage de maïs $(0,996$; tabl. 1), alors qu'il a été un peu infé- 
rieur avec I'herbe $(0,952 ; P<0,05)$. Il nous paraît cependant souhaitable d'estimer les quantités ingérées et la digestibilité sur la base d'un TRp égal à 1. En effet, la récupération incomplète du marqueur avec l'herbe semble due à une collecte difficile des fèces du fait de leur fluidité importante.

TABL. 1. - Estimation des quantités ingérées et de la digestibilité par la méthode à l'oxyde de chrome calculées à partir d'une teneur moyenne en chrome du concentré et d'une récupération totale du chrome (moyenne \pm écart-type).

\begin{tabular}{lccc}
\hline & Mais (essai 1A) & Maïs (essai 1B) & Herbe (essai 2) \\
\hline $\begin{array}{l}\text { Nombre de données } \\
\text { Taux de récupération (TRp) }\end{array}$ & 13 & 13 & 24 \\
$\begin{array}{l}\text { Quantité ingérée } \\
\text { (kg MO/j) }\end{array}$ & $1,01 \pm 0,05$ & $0,99 \pm 0,03$ & $0,95 \pm 0,06$ \\
estimée & $15,7 \pm 1,3$ & $16,3 \pm 2,9$ & $12,5 \pm 1,8$ \\
$\begin{array}{l}\text { Digestibilité } \\
\text { de la MO (\%) }\end{array}$ & $15,6 \pm 1,4$ & $16,5 \pm 3,3$ & $13,3 \pm \pm 1,9$ \\
mesurée & $0,69 \pm 0,02$ & $0,69 \pm 0,02$ & $0,79 \pm 0,04$ \\
estimée & $0,69 \pm 0,02$ & $0,69 \pm 0,03$ & $0,77 \pm 0,05$ \\
\hline
\end{tabular}

Pour les mêmes raisons, la variabilité du TRp est plus élevée avec l'herbe $(\mathrm{CV}=5,8 \%)$ qu'avec l'ensilage de maïs $(4,1 \%)$ où elle est semblable à celle obtenue avec du chrome mordancé (Chamberlain et Thomas, 1983). Cette erreur de mesure se traduit par un accroissement de la variabilité individuelle de 0,9 à 1,2 points pour l'estimation de dMO et de 0,5 à $0,7 \mathrm{~kg}$ de $\mathrm{MO} / \mathrm{j}$ dans celle de $\mathrm{Q}$.

Les différences individuelles du TRp n'étant pas significatives, la comparaison entre animaux n'est pas biaisée. La variabilité entre périodes a été élevée $(\mathrm{CV}=7,1 \%)$. Ces variations peuvent être attribuée d'une part à une adaptation insuffisante des animaux aux conditions de la mesure, d'autre part à l'imprécision de la détermination de la quantité de chrome ingérée due aux difficultés d'analyse de la teneur en chrome du concentré. En effet, l'obtention d'un sous échantillon broyé homogène étant difficile et la prise d'essai faible $(0,5 \mathrm{~g})$, la moyenne des 6 dosages réalisés pour chaque échantillon reste imprécise. De ce fait, il nous paraît préférable d'utiliser une teneur moyenne en chrome pour l'ensemble des périodes utilisant des concentrés issus d'un même lot de fabrication.

L'incertitude habituelle de l'estimation de l'ingestibilité ou de la digestibilité d'une ration, appréciée à titre d'exemple pour un lot de 6 vaches en collecte totale, sera accrue respectivement de $0,3 \mathrm{~kg} / \mathrm{j}$ de $\mathrm{MO}$ et 0,5 points du fait de la variabilité du taux de récupération. Les résultats obtenus ne seront pas biaisés si la quantité de chrome ingérée est déterminée avec précision.

Chamberlain D. G., Thomas P. C., 1983. Anim. Prod., 36, 155-157.

Poncet C., Rayssiguier Y., 1980. J. anim. Sci., 51, 180-185.

Thill N., François E., Thewis A., Thielemans M. F., 1978. Ann. Zootech., 27, 363-376. 